\title{
Pequenos para idade gestacional: fator de risco para mortalidade neonatal
}

\section{Small for gestational age: risk factor for neonatal mortality}

Márcia Furquim de Almeida e Maria Helena Prado de Mello Jorge

Departamento de Epidemiologia da Faculdade de Saúde Pública da Universidade de São Paulo. São Paulo, SP-Brasil 


\title{
Pequenos para idade gestacional: fator de risco para mortalidade neonatal
}

\section{Small for gestational age: risk factor for neonatal mortality}

\author{
Márcia Furquim de Almeida e Maria Helena Prado de Mello Jorge \\ Departamento de Epidemiologia da Faculdade de Saúde Pública da U niversidade de São Paulo. São \\ Paulo, SP-Brasil
}

\begin{abstract}
Resumo
Objetivo Estudar as variáveis contempladas na Declaração de Nascimento (DN) como possíveis fatores de risco para nascimentos pequenos para a idade gestacional (PIG) e o retardo de crescimento intra-uterino como fator de risco para a mortalidade neonatal.

Material e Método

As variáveis existentes na DN foram obtidas diretamente de prontuários hospitalares. Os dados referem-se a uma coorte de nascimentos obtida por meio da vinculação das declarações de nascimento e óbito, correspondendo a 2.251 nascimentos vivos hospitalares, de mães residentes, ocorridos no Município de Santo André, Região Metropolitana de São Paulo, no período de 1/1 a 30/6/1992, e aos óbitos neonatais verificados nessa coorte.

Resultados

Obteve-se a proporção de 4,3\% de nascimentos PIG, significativamente maior entre os recém-nascidos de pré-termo e pós-termo, entre os nascimentos cujas mães tinham mais de 35 anos de idade e grau de instrução inferior ao primeiro grau completo. Os recém-nascidos PIG apresentam maior risco de morte neonatal que aqueles que não apresentavam sinais de retardo de crescimento intra-uterino.

Conclusões $\quad$ Em áreas com menor freqüência de baixo peso ao nascer, é importante investigar a presença de retardo de crescimento intra-uterino entre os nascimentos prematuros e não apenas nos nascimentos de termo. O registro da data da última menstruação (ou da idade gestacional em semanas não agregadas na DN) facilitaria a detecção de PIGs na população de recém-nascidos.

Mortalidade neonatal [Saúde Pública]. Recém-nascido pequeno para a idade gestacional. Fatores de risco.
\end{abstract}

Correspondência para/Correspondence to: Márcia Furquim de Almeida - Av. Dr. Arnaldo, 715 - 01246-904 São Paulo, SP - Brasil. E-mail:marfural@usp.br

Edição subvencionada pela FAPESP (Processo no 97/09815-2).

Recebido em 13.11.1996. Reapresentado em 13.11.1997. Aprovado em 2.12.1997. 


\begin{abstract}
Introduction

Material and Method

Results

Conclusions

Variables of birth certificates were analysed as risk factors of SGA (Small for Gestational Age) infantis, and with a view to discovering if retarded intra-uterine growth was a risk factor neonatal mortality.

Data were obtained directly from 11 hospital medical records. A cohort of 2.251 hospital live births was obtained. Linkage of the death and birth certificates was undertaken to identify the neonatal deaths. The study was carried out in Santo André county in the S. Paulo Metropolitan area in the period from 1/1 to $30 / 6 / 1992$.

There were $4.3 \%$ of SGA live births. A higher statistically significant proportion of SGA was found in pre-term and post-term live births, among live births of mothers with 35 years of age and over and in those whose mothers had less than complete primary education. The SGA live births showed a higher risk of neonatal death, even when allowing for gestational age.

In areas where there is a low proportion of low birthweight, the presence of retarded intra-uterine growth may be an important risk factor to pre-term live births, due to the association between the pre-term and SGA. It would be easier to evaluate signs of retarded intra-uterine growth in the live birth population, if the gestational age data were registered, on the birth certificate in weeks.
\end{abstract}

Neonatal mortality [Public Health]. Infant, small for gestational age. Risk factors.

\section{INTRO DUÇÃO}

Desde meados dos anos 70 vem se observando um declínio da mortalidade infantil no País sendo que, em várias regiões, a mortalidade neonatal já é seu principal componente. No Estado de São Paulo, o coeficiente de mortalidade infantil era de 25,3 por mil nascidos vivos, em 1994, e os óbitos neonatais representavam cerca de $63,3 \%$ do total de mortes de menores de um ano $^{14}$. Assim, o estudo dos possíveis fatores de risco dessa mortalidade cresce em importância.

Vários autores ${ }^{5,22,25,27}$ mostram que o crescimento fetal intra-uterino retardado, que resulta em nascidos vivos considerados "pequenos para idade gestacional" (PIGs), constitui-se em importante fator de risco para a mortalidade neonatal . Por sua vez, os fatores de risco associados ao retardo do crescimento fetal intra-uterino são: pequeno ganho de peso materno durante a gestação, baixo peso materno anterior à gestação, baixa estatura da mãe, os quais são considerados como indicadores de possível presença de desnutrição materna ${ }^{1,7}$. Alguns estudos evidenciam que pode existir uma maior frequiência de PIGs em mães com idades inferiores a 20 anos e superiores a $35 \operatorname{anos}^{13}$ e em primíparas ${ }^{7,15}$ ou grandes multíparas ${ }^{8}$, bem como em produtos de gestações multíplas ${ }^{15}$. A freqüência de PIGs tem sido ain- da associada ao baixo nível de renda e menor grau de instrução da mãe ${ }^{1,7}$,esforço excessivo da mãe durante a gestação, hábito de fumar e consumo de drogas ${ }^{8}$. Verifica-se também retardo de crescimento intrauterino na presença de afecções maternas como hipertensão e infecções gênito-urinárias ${ }^{24}$, sendo que as anomalias congênitas também se constituem em fator predisponente para a maior freqüência de $\mathrm{PIGs}^{21}$.

Entre os estudos recentes sobre a freqüência de nascimentos com retardado de crescimento intrauterino, no Brasil, destacam-se os de Bertagnon ${ }^{10} \mathrm{e}$ de Ragonesi e col. ${ }^{23}$, realizados em hospitais de referência no Município de São Paulo, e o de Barros e col. ${ }^{7}$, levado a efeito em Pelotas, com base em dados populacionais. Alguns autores ${ }^{10,21}$ fazem distinção entre os PIGs de termo e pré-termo e mostram que os primeiros apresentam maior mortalidade e morbidade neonatais.

A definição mais aceita de pequenos para a idade gestacional é a que considera PIG como os nascidos vivos que apresentam valor inferior ao percentil 10 de peso ao nascer segundo idade gestacional ${ }^{18}$. Entretanto, a proporção de recém-nascidos considerados como PIG vai depender da curva de peso ao nascer usada como referência, sendo que estas curvas apresentam resultados distintos, dependendo das características da população e das possíveis exclusões realizadas nas amostras em estudo. Alguns autores ${ }^{19,25}$ 
mostram que a utilização dos dados da curva de Lubchenco e col. ${ }^{18}$ pode levar a uma subestimação da proporção do PIGs devido a fatores de ordem ambiental, como a elevada altitude de Denver, onde foi realizado esse citado estudo, e também a não exclusão de certos recém-nascidos, com características que, hoje, se sabe afetam o crescimento intrauterino, como por exemplo a presença de hipertensão materna, hábito de fumar e o baixo nível socioeconômico da população considerada.

Há outras curvas de crescimento com dados da população brasileira ${ }^{11,19}$, cujos valores de referência se iniciam com recém-nascidos de 29 semanas de idade gestacional. Sua utilização traria ganhos no sentido de maior comparabilidade quanto à composição da população; porém, ao utilizá-las seriam excluídos recém-nascidos de menor idade gestacional que podem apresentar retardo do crescimento intrauterino e, consequentemente, poderia subestimar a importância desse fator de risco de mortalidade neonatal. A curva de Garcia e col. ${ }^{15}$, obtida no Chile, traria também a vantagem de maior comparabilidade da população de referência, sendo que, desta, foram excluídos os recém-nascidos de mães com afecções crônicas, diabetes, cardiopatias, hipertensão e infecções virais ou parasitárias, as primíparas e os casos de gestações múltiplas. Os dados obtidos incluem nascimentos a partir da $26^{\text {a }}$ semana da gestação; porém, observa-se que o universo utilizado por esses autores para os recém-nascidos com idade gestacional entre 26 a 30 semanas era inferior a 10 indivíduos em cada uma das categorias. Esse pequeno número de casos gerou elevados desvios-padrão de peso ao nascer nesse grupo de nascimentos.

Dessa forma, a utilização da curva de Lubchenco e col. ${ }^{18}$ como referência, que permite a identificação de nascimentos PIG a partir da $24^{a}$ semana de gestação, ainda parece ser a mais adequada para o estudo da influência do retardo de crescimento intra-uterino sobre a mortalidade neonatal, mesmo sabendo que esta pode subestimar a proporção de PIGs.

A obtenção de dados populacionais sobre a freqüência de PIGs ainda não é viável rotineiramente no Brasil. O Sistema de Informações sobre Nascidos Vivos (SINASC) ${ }^{20}$, recentemente criado, permite conhecer a distribuição do peso ao nascer e da duração da gestação em nível populacional; porém, a informação sobre a idade gestacional encontra-se agrupada em categorias, não possibilitando identificar os nascimentos que se encontram abaixo do percentil 10 para cada semana da gestação.

Em 1993, foi realizada pesquisa que tinha por objetivo avaliar a fidedignidade das informações registradas na Declaração de Nascido Vivo (DN), documento de coleta de dados do SINASC ${ }^{20}$. A avaliação foi feita por meio da comparação dos dados registrados com aqueles existentes nos prontuários médicos, sendo que, para validar a variável duração da gestação, obteve-se a data da última menstruação da mãe. Fez parte ainda desse projeto de pesquisa a realização de estudo sobre mortalidade neonatal por meio da utilização da técnica de "linkage" dos sistemas de informação de nascido vivo e mortalidade ${ }^{4}$. A partir dos dados dessas duas pesquisas, foi possível obter uma coorte de nascidos vivos hospitalares para os quais se dispunham das informações sobre o peso ao nascer segundo idade gestacional, semana a semana, tornando factível estimar a prevalência de PIGs e permitindo, assim, conhecer a importância do crescimento fetal intra-uterino retardado como fator de risco da mortalidade neonatal .

O presente trabalho tem por objetivo avaliar as variáveis registradas na DN como possíveis fatores de risco para nascidos vivos PIGs e verificar sua probabilidade de morte .

\section{MATERIAL E MÉTO DO}

Foram estudados os nascimentos vivos hospitalares de mães residentes no Município de Santo André (São Paulo), no período de $1 / 1 / 92$ a 30/6/92, e os óbitos neonatais (menores de 28 dias) derivados desse conjunto de nascimentos, correspondendo às mortes ocorridas entre 1/1/92 a 27/7/92, no Município de Santo André ou outros municípios do Estado.

Partiu-se, inicialmente, de uma coorte de 3.175 nascidos vivos hospitalares, na qual tinha sido verificada a ocorrência de 55 óbitos neonatais, por meio da vinculação entre a Declaração de Óbito (DO)e a $\mathrm{DN}^{3,4}$.

As informações sobre peso ao nascer e duração da gestação foram retiradas diretamente dos prontuários hospitalares. A idade gestacional foi obtida a partir da data da última menstruação das mães, sendo excluídos do estudo todos os nascimentos para os quais não foi possível obter essas informações. Foram eliminados ainda 32 nascidos vivos resultantes de gestações múltiplas ou para os quais não havia informação sobre o tipo de gestação.

Para a obtenção do recém-nascidos considerados como PIGs utilizou-se a curva de peso ao nascer segundo a idade gestacional de Lubchenco ${ }^{18}$, considerando-se os que se situavam abaixo do percentil $1^{10}$. Esse citado estudo apresenta a distribuição de peso ao nascer para nascidos vivos com idade gestacional de 24 a 42 semanas, razão pela qual foram excluídos os casos cuja duração da gestação estava acima ou abaixo desse intervalo.

Após esses procedimentos, obteve-se uma coorte de 2.251 nascidos vivos hospitalares, na qual haviam ocorrido 48 óbitos neonatais. Apesar de se conhecer que as ano- 
malias congênitas podem ser um fator de risco para o retardo do crescimento intra-uterino ${ }^{21}$, no presente estudo não foram excluídos os nascimentos vivos que apresentavam essas anomalias, pois esta informação não consta da DN e o trabalho baseou-se na verificação das variáveis nela existentes e nos respectivos prontuários hospitalares.

A variável "idade gestacional" foi agregada em cinco categorias: 24 a 27 semanas; 28 a 31 semanas; 32 a 36 semanas; 37 a 41 semanas e 42 semanas e, posteriormente, agrupada em: gestações de "pré-termo" ( menos de 37 semanas) e "não pré-termo" (37 a 42 semanas). As variáveis "número de filhos anteriores ao do parto atual", "idade" e "grau de instrução da mãe" foram obtidas também dos prontuários médicos. A idade da mãe foi agregada em: menores de 20 anos; 20 a 34 anos e 35 anos e mais . A variável número de filhos anteriores foi categorizada em: mães nulíparas (nenhum filho), multíparas ( 1 a 4 filhos) e grandes multíparas (5 filhos ou mais). O grau de instrução da mãe foi agrupado em " inferior ao primeiro grau completo" e "primeiro grau completo e mais".

A probabilidade de morte foi definida como sendo a relação entre o número de óbitos neonatais e nascidos vivos X 1.000; obteve-se também o risco relativo para os recém-nascidos PIG e não PIG, calculando-se um intervalo de confiança de $95 \%$ e um valor de $\mathrm{p}<0,05$.

\section{RESU LTADO S E DISCU SSÃO}

A proporção de recém-nascidos considerados como PIGs no Município de Santo André foi de 4,3\%, valor próximo ao encontrado em estudo realizado no Município de São Paulo onde a freqüência foi de $4,9 \%{ }^{23}$. A comparação destes resultados, no entanto, deve ser cuidadosa, pois a população de estudo em São Paulo foi constituída por clientela usuária de um hospital universitário que, em geral, apresenta uma proporção mais elevada de baixa renda, na qual se encontra uma maior freqüência de PIGs; porém, como esse hospital se localiza em distrito com elevada qualidade de vida ${ }^{28}$, pode não ser representativa da clientela comumente usuária deste tipo de serviço. Por outro lado, o valor encontrado é bem inferior àquele obtido em estudo populacional realizado em Pelotas, em $1982^{7}$, onde a freqüência de PIGs foi de 7,2\%, bem como ao verificado em hospitais públicos da cidade do México, em $1982^{6}$, onde havia 9,8\% de nascidos vivos PIGs.

A menor prevalência de PIGs em Santo André, quando comparada à de Pelotas e Cidade do México, é reflexo da melhor situação socioeconômica da população dessa área, onde possivelmente existe menor freqüência dos fatores de risco que levam a nascimentos com retardo do crescimento intrauterino. O Município de Santo André, em estudo realizado pelo IBGE/UNICEF ${ }^{29}$, com base em dados do censo demográfico de 1991 e relativamente a condições de sobrevivência das crianças entre 0 e 6 anos de idade, ficou em $23^{\circ}$ lugar entre os $4.479 \mathrm{mu}-$ nicípios brasileiros estudados. Essa área apresentou, em 1992, uma freqüência de baixo peso ao nascer igual a 6,8\%, inferior, portanto, à da cidade de Nova York, que foi de 7,2\%, no mesmo ano. Hipótese semelhante pode ser aventada para a menor prevalência encontrada na população da Suécia ${ }^{17}$ onde os PIGs representaram 2\% do total de nascimentos, em 1987.

A Tabela 1 mostra que, à semelhança do estudo de Pelotas ${ }^{7}$, não foi encontrada associação entre a ordem de nascimento e a freqüência de PIGs, em Santo André. Esses resultados são discordantes de alguns trabalhos que evidenciam existir associação entre nascimentos de mães primíparas e a maior freqüência de $\mathrm{PIGs}^{1,8,15}$.

Também não se encontrou risco relativo estatisticamente significativo para a maior freqüência de PIGs entre as mães jovens (Tabela 2), resultado que é discordante do verificado por alguns autores ${ }^{1,7,23}$, que apontam a baixa idade materna como fator de risco para o retardo do crescimento intra-uterino.

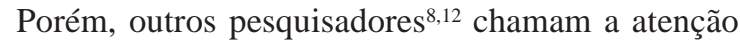
para o fato de haver resultados contraditórios na literatura, sugerindo que apenas em mães muito jovens poder-se-ia esperar um efeito isolado dessa variável sobre o retardo do crescimento intra-uterino; apontam ainda que vários estudos podem ter encon-

Tabela 1 - Nascidos vivos PIG (pequenos para idade gestacional) e não PIG e risco relativo (RR) segundo o número de filhos anteriores da mãe, Santo André, 1992.

\begin{tabular}{|c|c|c|c|c|c|c|c|c|c|c|}
\hline \multirow{2}{*}{$\begin{array}{l}\mathrm{N} \text { - } \text { filhos } \\
\text { anteriores }\end{array}$} & \multicolumn{2}{|c|}{ PIG } & \multicolumn{2}{|c|}{ Não PIG } & \multicolumn{2}{|c|}{ Total } & \multirow[t]{2}{*}{ RR } & \multirow[t]{2}{*}{ IC 95\% } & \multirow[t]{2}{*}{$\mathrm{X}^{2}$} & \multirow[t]{2}{*}{$P$} \\
\hline & $\mathrm{N} N$ & $\%$ & № & $\%$ & $\mathrm{~N} \cong$ & $\%$ & & & & \\
\hline Nenhum & 41 & 4,1 & 948 & 95,9 & 989 & 100,0 & 1,1 & $0,7-1,6$ & 0,06 & 0,80 \\
\hline 1 a 4 & 43 & 3,9 & 1.052 & 96,1 & 1.095 & 100,0 & 1,0 & & & \\
\hline 5 e mais & 5 & 8,1 & 57 & 91,9 & 62 & 100,0 & 2,1 & $0,8-5,0$ & 2,53 & 0,11 \\
\hline Total & 89 & 4,1 & 2.057 & 95,9 & 2.146 & 100,0 & & & & \\
\hline
\end{tabular}

Nota - Excluídos os nascidos vivos sem informação sobre o número de filhos anteriores da mãe.

$X^{2}=2,5 ; 2$ graus de liberdade; $p=0,28$

IC - Intervalo de confiança. 
trado associação entre a baixa idade materna e nascimentos PIG, devido à presença de fatores de confusão, tais como, consumo de cigarros durante a gestação, baixo grau de instrução e de renda das mães desse grupo etário.

Por outro lado, observou-se risco significativamente maior de frequiência de nascidos vivos PIGs entre as mulheres de 35 anos e mais (Tabela 2). Esta variável pode estar expressando o efeito, não apenas da avançada idade materna, mas de gestações sucessivas com pequeno intervalo interpartal, que também se constitui em fator de risco para recém-nascidos $\mathrm{PIGs}^{8}$.A associação encontrada entre a elevada idade materna e a presença de PIGs pode, em alguma medida, estar expressando a incorporação, em Santo André, de uma tendência já observada em outros países, que é o retardo do início do processo reprodutivo em mulheres com maior grau de escolaridade, grupo no qual se encontra uma associação positiva entre essas variáveis ${ }^{8}$.

O baixo nível de instrução materna pode ser considerado como fator de risco para nascimentos com retardo de crescimento intra-uterino; verificou-se que as mães com grau de instrução inferior ao primeiro grau apresentaram uma chance $70 \%$ superior à das que tinham o primeiro grau completo ou mais de instrução (Tabela 3). Entre as mães com nível universitário, que representaram $9,0 \%$ da população, não se encontrou nenhum nascimento com retardo do crescimento intra-uterino.

Os resultados obtidos mostram que há uma maior proporção de PIGs tanto com a diminuição da idade gestacional como nos nascimentos de pós-termo, observando-se, na Tabela 4, um gradiente quando se toma como referência a proporção de PIGs entre os nascidos vivos de gestação a termo. Esses valores sugerem que o retardo do crescimento intra-uterino pode vir a ser um fator de risco para a prematuridade ${ }^{24}$. Alguns autores observaram que há fatores de risco que são comuns tanto para o retardo do crescimento intra-uterino como para a prematuridade, a exemplo da presença de infecções gênito-urinárias maternas ${ }^{24,26}$ e hipertensão na gravidez. Berkowitz e Papiernick ${ }^{9}$ indicam que a prematuridade e o crescimento fetal retardado constituem-se processos fisiopatológicos distintos, haven-

Tabela 2 - Nascidos vivos PIG e não PIG e RR segundo a idade da mãe, Santo André, 1992.

\begin{tabular}{lrrrrrrrrrr}
\hline \multirow{2}{*}{ Idade } & \multicolumn{2}{c}{ PIG } & \multicolumn{2}{c}{ Não PIG } & \multicolumn{2}{c}{ Total } & RR & IC $95 \%$ & $X^{2}$ & P \\
& No & $\%$ & No & \multicolumn{1}{c}{$\%$} & No & $\%$ & & & & \\
\hline M enos de 20 & 25 & 5,2 & 455 & 94,8 & 480 & 100,0 & 1,5 & $0,9-2,3$ & 2,51 & 0,11 \\
20 a 34 & 56 & 3,6 & 1.053 & 96,4 & 1.559 & 100,0 & 1,0 & & \\
35 e mais & 14 & 7,1 & 183 & 92,9 & 197 & 100,0 & 2,1 & $1,1-3,5$ & 5,64 & 0,01 \\
\hline Total & 95 & 4,2 & 2.141 & 95,8 & 2.236 & 100,0 & & & \\
\hline Nota - Excluídos os nascidos vivos sem informação sobre idade da mãe.
\end{tabular}

$X^{2}=6,7 ; 2$ graus de liberdade; $p=0,03$

Tabela 3 - Nascidos vivos PIG e não PIG e risco relativo (RR) segundo grau de instrução da mãe, Santo André, 1992.

\begin{tabular}{|c|c|c|c|c|c|c|c|c|c|c|}
\hline \multirow{2}{*}{$\begin{array}{l}\text { Grau de } \\
\text { instrução }\end{array}$} & \multicolumn{2}{|c|}{ PIG } & \multicolumn{2}{|c|}{ Não PIG } & \multicolumn{2}{|r|}{ Total } & \multirow[t]{2}{*}{$\mathrm{RR}$} & \multirow[t]{2}{*}{ IC 95\% } & \multirow[t]{2}{*}{$\overline{X^{2}}$} & \multirow[t]{2}{*}{$\bar{P}$} \\
\hline & № & $\%$ & № & $\%$ & $\mathrm{No}$ & $\%$ & & & & \\
\hline Inferior ao primeiro grau completo & 41 & 5,3 & 729 & 94,7 & 770 & 100,0 & 1,8 & $1,1-2,9$ & 4,8 & 0,02 \\
\hline Primeiro grau completo e mais & 22 & 3,0 & 702 & 97,0 & 724 & 100,0 & - & - & - & - \\
\hline Total & 63 & 4,2 & 1.431 & 95,8 & 1.494 & 100,0 & & & & \\
\hline
\end{tabular}

Nota - excluídos os nascidos vivos sem informação sobre grau de instrução da mãe.

Tabela 4 - Nascidos vivos PIG e não PIG e risco relativo (RR) segundo duração da gestação, Santo André, 1992.

\begin{tabular}{|c|c|c|c|c|c|c|c|c|c|c|}
\hline \multirow{2}{*}{$\begin{array}{l}\text { Duração } \\
\text { gestação } \\
\text { (semanas) }\end{array}$} & \multicolumn{2}{|c|}{$P I G$} & \multirow{2}{*}{$\begin{array}{l}\text { N ão } \\
\text { № }\end{array}$} & PIG & \multicolumn{2}{|c|}{ Total } & \multirow[t]{2}{*}{$\mathrm{RR}$} & \multirow[t]{2}{*}{ IC 95\% } & \multirow[t]{2}{*}{$x^{2}$} & \multirow[t]{2}{*}{ P } \\
\hline & № & $\%$ & & $\%$ & № & $\%$ & & & & \\
\hline $24-27$ & 3 & 15,0 & 17 & 85,0 & 20 & 100,0 & 4,0 & $1,4-11,6$ & 6,70 & 0,009 \\
\hline $\begin{array}{l}28-31 \\
32-36\end{array}$ & $\begin{array}{l}4 \\
9\end{array}$ & $\begin{array}{r}10,5 \\
5,0\end{array}$ & $\begin{array}{r}34 \\
170\end{array}$ & $\begin{array}{l}89,5 \\
95,0\end{array}$ & $\begin{array}{r}38 \\
179\end{array}$ & $\begin{array}{l}100,0 \\
100,0\end{array}$ & $\begin{array}{l}2,8 \\
1,3\end{array}$ & $\begin{array}{l}1,1-7,2 \\
0,7-2,6\end{array}$ & $\begin{array}{l}4,55 \\
0,70\end{array}$ & $\begin{array}{r}0,03 \\
0,4\end{array}$ \\
\hline $37-41$ & 73 & 3,8 & 1.865 & 96,2 & 1.938 & 100,0 & 1,0 & & & \\
\hline 42 & 8 & 10,5 & 68 & 89,5 & 76 & 100,0 & 2,8 & $1,4-5,6$ & 8,66 & 0,003 \\
\hline Total & 97 & 4,3 & 2.154 & 95,7 & 2.251 & 100,0 & & & & \\
\hline
\end{tabular}

$X^{2}=17,84 ; 4$ graus de liberdade; $p=0,001$ 
do, porém, uma sobreposição de fatores de risco para ambos resultando, dessa forma, em elevadas proporções de nascimentos de pré-termo com retardo de crescimento intra-uterino.

Kramer ${ }^{16}$ chama a atenção para o fato de que nos países do primeiro mundo a principal causa do baixo peso ao nascer é a prematuridade e, nos menos desenvolvidos, o retardo do crescimento intrauterino. Os dados obtidos sugerem que, em áreas como Santo André, onde a proporção de nascimentos com baixo peso não é tão elevada $(6,8 \%)$, a presença da prematuridade deve ser levada em conta como fator importante na etiologia do retardo do crescimento intra-uterino e do baixo peso ao nascer.

Com relação à mortalidade neonatal, verificouse que, no conjunto da coorte, a probabilidade de morte neonatal foi de 21,3 por mil nascidos vivos (Tabela 5). Este valor é superior àquele encontrado no estudo que utilizou a coorte original de nascimentos vivos em Santo André, que foi de 17,1 por mil nascidos vivos ${ }^{3,4}$, fato que se deve à exclusão dos nascimentos múltiplos e daqueles que não tinham informação sobre peso ao nascer e data da última menstruação registrados nos prontuários hospitalares.

Verificou-se que os recém-nascidos PIG apresentaram um risco de morte significativamente maior que aqueles não PIG (Tabela 5), indicando que a presença de retardo de crescimento intra-uterino constitui-se em fator de risco para a mortalidade neonatal.

Ao se trabalhar com a idade gestacional em semanas desagregadas, foi possível verificar que não houve sobreviventes entre recém-nascidos com idade gestacional inferior a 25 semanas e com peso ao nascer menor que 900 gr. Comparando os presentes resultados com estudos realizados em países europeus, no período de 1983 a $1986^{30}$,verifica-se que, em 1992, os nascimentos de Santo André apresentaram comportamento semelhante ao encontrado na Holanda e Alemanha, na década de 80, evidenciando ainda que localidades como Inglaterra e Escócia já apresentavam, nesse período, sobreviventes com 24 semanas de gestação. A expansão dos cuidados neonatais intensivos permitiu o aumento da sobrevivência de recém-nascidos de baixa idade gestacional, como mostraram Allen e col. ${ }^{2}$ que encontraram sobreviventes com 23 semanas de idade gestacional e de peso ao nascer em torno de 600 gr., em estudo realizado na década de 90, nos Estados Unidos.

Como foi apresentado anteriormente, encontrouse maior proporção de PIGs entre os recém-nascidos de pré-termo; ao se ajustar o risco de morte neonatal para a presença de prematuridade, ainda assim, observa-se risco maior para os nascidos vivos com retardo de crescimento intra-uterino

Verifica-se também elevado risco de morte para os nascimentos que são, ao mesmo tempo, prematuros e com retardo de crescimento intra-uterino, sendo que menos da metade desses recém-nascidos sobreviveram ao período neonatal (Tabela 6).

Nota-se ainda que os PIGs de pré-termo têm uma chance de morrer no período neonatal 120,8 vezes a dos nascidos vivos de gestação a termo e sem retardo de crescimento intra-uterino (considerandose um intervalo de confiança de $95 \%$, risco relativo entre 55,3 a 264,7 com um $\mathrm{X}^{2}$ de 539,68 e p menor que 0,01 ).

Almeida, $1995^{3}$, utilizando o critério de PIG simplificado (nascidos vivos de termo com peso inferi-

Tabela 5 - Estimativa da probabilidade de morte (q) e RR dos nascidos vivos PIG e não PIG, Santo André, 1992.

\begin{tabular}{|c|c|c|c|c|c|c|c|c|c|}
\hline $\mathrm{N}$ ascidos & vivos & Ó bito & Sobrevivente & Total & $q$ & $\mathrm{RR}$ & IC $95 \%$ & $x^{2}$ & $P$ \\
\hline PIG & & 13 & 84 & 97 & 134,0 & 8,3 & $4,5-15,1$ & 61,69 & 0,0000 \\
\hline Não PIG & & 35 & 2.119 & 2.154 & 16,2 & 1,0 & & & \\
\hline Total & & 48 & 2.203 & 2.251 & 21,3 & & & & \\
\hline
\end{tabular}

Tabela 6 - Estimativas da probabilidade de morte $(q)$ e RR dos nascidos vivos PIG e não PIG segundo duração da gestação, Santo André, 1992.

\begin{tabular}{|c|c|c|c|c|c|c|c|c|}
\hline $\begin{array}{l}\text { Duração } \\
\text { gestação }\end{array}$ & Ó bito & Sobrevivente & Total & $\begin{array}{r}q \\
\times 1.000\end{array}$ & $\mathrm{RR}$ & IC $95 \%$ & $x^{2}$ & $\mathrm{P}$ \\
\hline \multicolumn{9}{|l|}{ Pré-termo } \\
\hline PIG & 9 & 7 & 16 & 562,5 & 4,8 & $2,7-8,4$ & 23,46 & 0,0000 \\
\hline $\begin{array}{l}\mathrm{N} \text { ão PIG } \\
\mathrm{N} \text { ão pré-termo }\end{array}$ & 26 & 195 & 221 & 117,6 & 1,0 & & & \\
\hline PIG & 4 & 77 & 81 & 54,8 & 10,6 & $3,3-33,7$ & 24,25 & 0,0000 \\
\hline Não PIG & 9 & 1.924 & 1.933 & 4,6 & 1,0 & & & \\
\hline Total & 48 & 2.203 & 2.251 & 21,3 & & & & \\
\hline
\end{tabular}


or a $2.500 \mathrm{~g}$ ) não encontrou risco de morte estatisticamente significativo para esse grupo de nascimentos. Os resultados obtidos sugerem que ao se utilizar critérios mais adequados para identificação de nascimentos PIG, estes constituem-se fator de risco para a mortalidade neonatal.

Em conclusão, os fatores de risco para o retardo do crescimento intrauterino foram as gestações de pré-termo e pós-termo, idade materna de 35 anos e mais, grau de instrução inferior ao primeiro grau completo. Observou-se uma tendência decrescente da freqüência de PIGs com aumento de idade gestacional até atingir as gestações de termo.

O retardo do crescimento intrauterino constituise em fator de risco para a mortalidade neonatal, tanto para recém-nascidos de pré-termo como de não prétermo. A utilização do conceito de PIG simplificado (recém-nascidos de termo com baixo peso ao nascer) pode subestimar a importância do retardo do crescimento intra-uterino como fator de risco da mortalidade neonatal.

Para que estudos dessa natureza passem a ser rotineiramente possíveis por meio dos dados dos sistemas oficiais de informação (Sistema de Informação de Mortalidade e SINASC), sugere-se que a variável duração de gestação deixe de ser registrada em classes pré-estabelecidas, tal como se apresenta hoje na DN, passando a ser anotada a idade gestacional (em semanas) de cada nascido vivo, de forma desagregada. A mesma conduta é sugerida para as DO, de modo a possibilitar a identificação dos recém-nascidos que apresentem sinais de retardo de crescimento intra-uterino, ao se utilizar essa fonte de dados.

\section{REFERÊNCIAS}

1. ABRAMS, B. \& NEWMAN, V. Small for gestacional age birth: maternal predictors and comparison with risk factors of spontaneous preterm delivery in the same cohort. Am. J. Obstet. Gynecol., 164: 785-90, 1991.

2. ALLEN, M.C. et al. The limit of viability neonatal outcome of infants born at 22 to 25 week's gestation. N. Engl. J. Med., 329: 1597-601, 1993.

3. ALMEIDA, M.F. Mortalidade neonatal em Santo André. São Paulo, 1995. [Tese de Doutorado - Faculdade de Saúde Pública da USP].

4. ALMEIDA, M.F. \& MELLO JORGE, M.H.P. O uso da técnica de "linkage" de sistemas de informação em estudos de coorte sobre mortalidade neonatal. Rev. Saúde Pública, 30: 141-7, 1996.

5. BAKKTEIG, L.S. \& MAGNUS, P. Small for gestationalage (SGA) definitions and associated risks. Int. J. Technol. Assess. Health Care, 8: 139-46, 1991.

6. BALCAZAR, H. \& HASS, J.D. Retarded fetal growth patterns and early neonatal mortality in a Mexico City population. Bull. Pan Am. Health Organ., 25: 55-63, 1991.

7. BARROS, F.C. et al. Comparison of the causes and consequences of prematurity and intrauterine growth retardation: a longitudinal study in southern Brazil. Pediatrics, 90: 238-44, 1992.

8. BERENDES, H. W. Maternal determinants of perinatal mortality of intrauterine growth retardation and preterm delivery. In: Baum, J.D. Birth risks. New York, Raven Press, 1993. p. 47-58.

9. BERKOWITZ, G. S. \& PAPIERNIK, E. Epidemiology of preterm birth. Epidemiol. Rev., 15: 414-43, 1993.
10. BERTAGNON, J. R. D. Recém-nascido pequeno para idade gestacional- algumas características epidemiológicas. São Paulo, 1991. [Dissertação de Mestrado - Faculdade de Saúde Pública da USP].

11. BRENELLI, M.A. \& MARTINS FILHO, J. Curvas de crescimento intrauterino da população de nascidos vivos na maternidade do Caism-Unicamp. J. Pediatr., 68: 21-5, 1992.

12. CANOSA, C.A. et al. Extremes of reproductive ages. In: Baum, J. D. Birth risks, K. New York, Raven Press, 1993. p. 189-202.

13. ESCAMILLA, R. P. \& POLLITT, E. Causes and consequences of intra-uterine growth retardation in Latin America. Bull. Pan. Am. Health Organ., 26: 128-47, 1992.

14. FUNDAÇÃO SEADE. Software- Perfil de saúde. São Paulo, 1996.

15. GARCIA, G. J. et al. Evaluación neonatal del crescimiento intrauterino. Rev. Latinoam. Perinatol., 13: 5-14, 1993.

16. KRAMER, M. S. et al. Impact of intrauterine growth retardation and body proportionality on fetal and neonatal outcome. Pediatrics, 86: 707-13, 1990.

17. LEIJON, I. The prognostic significance of antenatal diagnosis of fetal growth retardation. Int. J. Technol. Assess. Health Care, 8 (Suppl): 176-81, 1992.

18. LUBCHENCO, L.O. et al. Intrauterine growth as estimated from liveborn birth-weight data at 24 to 42 weeks of gestation. Pediatrics, 32: 793-800, 1963.

19. MARGOTTO, P. R. Curvas de crescimento intra-uterino: estudo de 4.413 recém-nascidos únicos de gestações normais. J. Pediatr., 71: 11-21, 1995 
20. MELLO JORGE, M. H. P. et al. Avaliação do sistema de informação sobre nascidos vivos e o uso de seus dados em epidemiologia e estatísticas de saúde. Rev. Saúde Pública, 27 (6, supl): 1-46, 1993.

21. OTT, W. J. Small for gestacional age fetus and neonatal outcome: reevalution of the relationship.Am. J. Perinatol., 12: 396-400, 1995.

22. PALTA, M. et al. The relation of maternal complications to outcomes in very low birthweight infants in an era of changing neonatal care. Am. J. Perinatol., 13: 109-14, 1996.

23. RAGONESI, S. M. A. et al. Crescimento intrauterino retardado. Rev. Latinoam. Perinatol., 13: 15-46, 1993.

24. SALAFIA, C. M. et al. The very low brithweight infant: maternal complications leading to preterm birth, placental lesions, and intrauterine growth. Am. J. Perinatol., 12: 106$10,1995$.

25. SATANDER, F. F. et al. Comparison of three standards for evaluating fetal growth. Bull. Pan Am. Health Organ., 26: 37-46, 1992.

26. SAVITZ, D. A. et al. Epidemiologic characteristics of preterm delivery: ethiologic heterogenity. Am. J. Obstet. Gynecol., 164: 46-71, 1991.

27. SOTO, I. C. et al. Valores de referencia para evaluar el crescimiento en nascimientos ocurridos en la ciudad de Mexico. Salud Publica Mex., 30: 68-80, 1988.

28. SPOSATTI, A. et al. Mapa da exclusão social da cidade de São Paulo. São Paulo, Núcleo de Estudos de Seguridade e Assistência Social PUC/SP, 1995.

29. UNICEF/IBGE. Municípios brasileiros: crianças e suas condições de sobrevivência. Rio de Janeiro, 1994.

30. WORKING GROUP ON VERY LOW BIRTH WEIGHT INFANTS- (WGVLBI). European Community colaborative study of outcome of pregnancy between 22 and 28 weeks gestation. Lancet, 336: 782-4, 1990. 